\title{
Health and disease: what can medicine do for philosophy?
}

\author{
J G Scadding University of London
}

\section{Author's abstract}

Philosophical discussions about health and disease often refer to a 'medical model' of bodily disease, in which diseases are regarded as causes of illness; diagnosis consists in identifying the disease affecting the patient, and this determines the appropriate treatment. This view is plausible only for diseases whose cause is known, though even in such instances the disease is the effect on the affected person, and must not be confused with its own cause. But in fact the medical diagnostic process which progresses from recognition of patterns of symptoms and signs, through search for abnormalities of structure and/or function, towards knowledge of causation of ten stops short of this desirable end-point; and at whatever point it comes to a halt, its result is expressed in terms of 'diseases'. Thus in medical discourse the names of diseases are a convenient device by which the current conclusion of the diagnostic process can be stated briefly; and they have widely varying factual implications. This nominalist analysis of the medical usages of the names of diseases has consequences for definitions of health and disease, and for some problems in medical ethics.

Reading Professor Hare's paper (1) based on his John Locke Lecture entitled What can philosophy do for medicine? , I was reminded of the comment made by a non-medical participant at an early conference on the application of computers to medical diagnosis that until the physician is willing to investigate his own terminology, all the mathematician can do is to stand in the wings and help out in minor ways (2). This stimulated me in 1967 to set out in terms which I hoped would be comprehensible to a non-medical enquirer the results of an analysis of the meaning of medical diagnostic statements with which as a practising clinician, mainly in the field of respiratory disease, I had been concerned for many years (3). Some psychiatrists have found it relevant to their special problems of nosology $(4,5,6)$. Their interest in an analysis of the semantic problems of medical diagnosis,

\section{Key words}

Disease; health; definition, nominalist and essentialist; medical diagnosis; psychiatric diagnosis; evaluation, subjective and objective. undertaken to clarify discussion of some respiratory diseases, encourages me to think that it may be relevant to some of the ethical problems in relation both to individual patients and to society which today are widely discussed. I therefore venture to outline this analysis, and then to comment on its possible relevance to these problems.

\section{The heterogeneity of 'diseases'}

Consideration of the usages of the names of diseases in medical discourse shows that they have widely differing factual implications. The simple idea that diseases are causes of illness and that diagnosis consists in identifying the disease which is causing the patient's illness is obviously erroneous, although it is implicit in colloquial usage. I will illustrate this by a series of hypothetical scenarios, all starting with a patient consulting a physician because he has a persistent cough.

1. The cough produces mucoid phlegm, and there are episodes of more severe symptoms after upper respiratory infections. The physician diagnoses chronic bronchitis.

The patient may take this to mean that his cough is caused by chronic bronchitis.

The physician should know that at present the most generally accepted definition of chronic bronchitis is in terms of clinical description, and thus it is absurd to regard this disease as the cause of the symptoms by which it is defined. He should be considering possible causal factors. The patient is likely to be a smoker; if so, the physician will probably tell him that cigarette smoking is the principal cause of his symptoms.

2 . Because the cough produces persistently yellow phlegm, further investigation is undertaken, and this shows that there is localised dilatation of some bronchi, leading to a diagnosis of bronchiectasis.

The patient is likely to think that this impressivelynamed demon is the real cause of his symptoms.

The physician should know that in saying 'bronchiectasis' he has only said 'bronchial dilatation' in Greek. In a few cases this is a consequence of a past episode of acute broncho-pulmonary inflammation from which the patient made an otherwise complete recovery, but in most it appears during the course of chronic broncho-pulmonary disease of various sorts; 
and the mechanisms which lead to dilatation of the bronchi are diverse. In themselves, dilated bronchi cause no symptoms; the contribution of infective and vascular changes in them to the production of symptoms varies greatly from case to case, and should be individually assessed. Thus, a diagnosis of bronchiectasis is a claim to knowledge that the patient has dilated bronchi, with the implication that at least some of the symptoms are due to changes in them. It conveys no necessary implication about the pathogenesis of this anatomical abnormality; if anything is known about this, a further diagnostic term is required to specify it.

3. The patient complains of episodes of shortness of breath and wheeze as well as cough, and tests of the ventilatory function of the lungs show wide variations in expiratory airflow resistance. This leads to a diagnosis of asthma.

Again, the patient will probably think that the cause of his symptoms has been identified.

The physician should know that in applying the diagnostic term 'asthma' he is claiming no more than that the wheezy breathlessness is due to wide variations in resistance to expiratory air-flow in the lungs. He may be able to show that the patient's bronchi are abnormally reactive to a variety of stimuli, but can only speculate about the causes of this abnormal reactivity. $\mathrm{He}$ will try to identify factors causing increases in resistance. In many instances, he will fail. If he succeeds, he may qualify 'asthma' by a term indicative of this factor. Thus in making an unqualified diagnosis of asthma, he is categorising the patient's case in a group defined by a specified abnormality of function.

4. Investigation of the coughing patient includes a chest $\mathrm{x}$-ray, which is abnormal, and tubercle bacilli are found in the sputum. The physician makes a diagnosis of pulmonary tuberculosis.

The patient will probably think of tuberculosis as the cause of his illness.

For the physician, the diagnosis of tuberculosis is a claim to knowledge that mycobacterial infection is the necessary cause of the abnormalities which he has found in the patient's body, and which he believes to be the cause of the symptoms; in current nosology, 'tuberculosis' refers to the effects of infection by mycobacteria on an animal host, although these effects are greatly dependent upon factors of host resistance (see appendix 1). Thus, he must not confuse the causal agent, the tubercle bacillus, with its effect, tuberculosis.

\section{Classes of defining characteristics for primary diagnostic categories}

These examples illustrate the four main classes of characteristics by which diseases can now be defined. I have chosen them from the respiratory field, in which I first became concerned with semantic problems in medicine $(7,8,9,10)$, but the analysis is generally applicable. In the order in which, historically, they entered medical practice, they are:

\section{i. CLINICAL DESCRIPTION (SYNDROME)}

Initially, diseases could be defined only by description. Patients with a recognisably similar pattern of symptoms and signs were said to be suffering from the same disease. A recognisable pattern of this sort is called a syndrome; the relationship between 'disease' and 'syndrome' is discussed below. Study of a group of patients recognised in this way will be directed towards discovery of any distinctive changes in structure or function and elucidation of causation. But even when causation is not known, and anatomical and functional changes are inconstant or not distinctive, clinical study will lead to knowledge of prognosis and may discover ways to ameliorate symptoms and possibly to improve prognosis. Thus, it is useful from the beginning to have a name by which to refer to the phenomena displayed by a clinically recognisable group of patients; and this is adopted as the name of a disease.

Most psychiatric diagnostic categories are still syndromally defined, and some categories definable only in this way remain in current use in nonpsychiatric medicine. For example, migraine refers to a sort of recurrent headache whose recognition is important in several respects. The headaches are distressing and may interfere with normal activities; and while migraine is not dangerous to life, headaches of similar character may be caused by serious organie changes in the brain, so that a positive diagnosis migraine is important. The description of the disease 'migraine' is based on the study of individuals who have recurrent headaches conforming to a carefully defined pattern, and in whom no structural abnormality of the nervous system is found. It includes such facts as the proportions of cases in which symptoms such as visual disturbances and vomiting precede or follow the headaches, the age- and sexincidence, the responses to various medicaments, and an account of evidence relevant to precipitating factors and to possible vascular mechanisms underlying the symptoms; but these findings, being inconstant, have no place in the definition.

\section{ii. DISORDER OF STRUCTURE (MORBID ANATOMY)}

Historically, when a disease defined by clinical $D$ description was found to be associated constantly with a recognisable morbid-anatomical change, it tended to be re-defined, implicitly or explicitly, and possibly renamed in these terms; and with the development of microscopy, in terms of microscopic structure (histology). As examples of diseases defined in these ways in current nosology, mitral stenosis and cirrhosis $Q$ of the liver may be quoted. The description of each of these diseases is based on the study of patients in whom ? it has been established that the relevant abnormality of structure is present, and is an account of all abnormalities found to be associated with it. If the same word is used to name both the structural abnormality and the disease, it is important to distinguish carefully between these two usages; as the 
name of a disease, 'mitral stenosis' refers, not to the narrowing of the mitral valve alone, but also to the symptoms and signs and changes in other organs that may be consequent upon it.

\section{iii. DISORDER OF FUNCTION (PATHOPHYSIOLOGY)}

Similarly, when a specifiable disorder of function was found to be associated with a recognisable clinical syndrome, it was likely to be used as the defining characteristic for an appropriately named diagnostic category. Many examples can be found in current nosology. They range from broad categories, such as hypertension, to deficient or excessive secretion of a single hormone by an endocrine gland. Again, it is important to distinguish between the connotations of a word when used to specify a disorder of function and when used to refer to the disease which is the consequence of this disorder; 'hypothyroidism' as the name of a disease refers, not to deficient secretion of thyroid hormones alone, but also to the consequences of this deficiency.

Diagnosis of a disease defined in morbid-anatomical or pathophysiological terms carries no necessary implications about the causation of the underlying abnormality of structure or function.

\section{iv. CAUSATION (AETIOLOGY)}

When the cause of a disease becomes known, the disease is generally re-defined, explicitly or implicitly, in causal terms. Tuberculosis, already mentioned, is an example of this. In medical discourse, 'influenza' should be used to refer only to the disease caused by an influenza virus, notwithstanding its general colloquial use to refer to any short-term febrile respiratory illness presumed to be caused by one of many possible viral and other agents. Although the common infectious fevers are nearly always diagnosed confidently on clinical grounds alone, the ultimate criterion of the correctness of the diagnosis for those whose causal agent has been identified is evidence of infection by this agent, and thus they should now be defined as the diseases caused by the relevant agents.

Groups ii, iii, and iv can be subdivided in an increasing number of ways. For instance, abnormalities of chromosome structure or number now define some genetically definable disorders, such as sub-types of Down's syndrome; biochemical abnormalities, possibly linked to single gene defects, now define some inherited diseases, previously syndromally-defined; and deficiencies of vitamins and other nutritional factors can be included in group iv as causal defining characteristics of diseases.

The advance of medical knowledge is, in general, towards causation; it often leads to re-definition of diseases, disorders of structure or of function displacing syndrome, and causation in turn displacing these as defining characteristics. It is thus inevitable that diagnostic terms of these various sorts co-exist in current nosology. Unless the differing factual implications of the names of diseases defined in these various ways are recognised, confusion is inevitable.

Another possible source of confusion is failure to recognise that when the basis of definition of a disease is changed, the set of patients specified by the new definition to provide the description of the disease is unlikely to be identical with that specified by the old; in other words, the disease defined on the new basis may not be identical with that defined on the old. For instance, myxoedema, defined syndromally, is not the same as hypothyroidism, defined functionally. And tuberculosis provides an excellent example of the effects of changes in defining characteristics with advances in knowledge (see appendix 1).

\section{Simple and compound diagnostic categories}

Another complication is that a disease may be defined by characteristics derived from more than one field of study. Such a disease should be regarded as the intersection of the sets constituted by two or more primary or simple categories, constituting a compound diagnostic category. This may be made obvious by the name used for it; for instance, pneumococcal pneumonia is the intersection of aetiologically and morbid-anatomically defined sets. It may be less obvious when an old-established name conceals the complexity of the definition entailed by current knowledge. For example, the definition of pernicious anaemia, originally described by Addison as a clinical syndrome, now requires elements referring to the abnormal morphology of the red blood cells and their precursors (megaloblastic anaemia) and to the functional disorder which leads to it (defective absorption of vitamin B12 due to gastric intrinsic factor deficiency).

\section{A note on definitions in scientific contexts}

In the foregoing analysis of current nosology, I have sought to make explicit the factual implications of medical usages of the names of diseases. This analysis is an essential preliminary to definitions of diseases in what Popper (11) calls a methodologically nominalist as opposed to an essentialist manner. Nominalist definitions do not attempt the impossible task of revealing the essence of the definiendum, but state how words or other symbols are to be related to observable phenomena. Popper points out that essentialist definitions, depending upon intuitive acceptance for their validity, have no place in science; although he claims that science could progress without definitions of any sort, he admits that this would be at the cost of excessive verbosity, and accepts nominalist definitions as convenient shorthand symbols. Indeed, it is difficult to see how problems in physics could be discussed without the universal acceptance of nominalist definitions of its concepts in terms of the dimensions length, mass, and time. Similarly, I do not see how a disease-terminology can be used in scientific discussion of medical problems without nominalist definitions of diseases. The name of a disease, as properly used in medical discourse, is a convenient 
brief statement of the current conclusion of a diagnostic process which for each patient follows the general direction of the historical advance of medicine from recognition of syndrome towards knowledge of causation but often stops short of this desirable endpoint.

\section{The medical concept 'a disease'}

Is the concept 'a disease' necessary in medical discourse? Important problems may be discussed, and decisions may be made, especially in emergency situations, without use of the disease terminology. Although its results are expressed in this terminology, one of the most successful applications of computing to diagnosis, that concerned with acute abdominal pain, is in effect an aid to the decision whether to operate or not (12). Moreover, opinion among doctors about whether words in common use for conditions which might lead to medical consultation refer to diseases varies, though less than among laymen (13). But though it seems possible that a medical language which dispensed with the names of diseases might be elaborated, the prospect of this must be remote, and it is certain that for the foreseeable future the concept 'a disease' will persist in medical as well as in colloquial discourse. It is therefore essential that the implications of medical usages of the names of diseases should be made explicit. The following statement attempts to do this, in terms which allow it to be applied in all biological contexts:

In medical discourse, the name of a disease refers to the sum of the abnormal phenomena displayed by a group of living organisms in association with a specified common characteristic or set of characteristics by which they differ from the norm of their species in such a way as to place them at a biological disadvantage.

This general statement recognises the logical heterogeneity of the concept 'a disease' in medical contexts. If it is accepted, a particular disease can be defined by specification of the common feature which characterises the group upon the study of which its description is based. This defining characteristic may be of several sorts: descriptive or syndromal, morbidanatomical, pathophysiological, and aetiological. As knowledge advances, syndrome tends to be displaced as the basis of definition by more objective features, among which, when possible, aetiology takes precedence.

\section{Qualitative and quantitative elements in definition}

The first step in definition is qualitative. What sort of deviation from the norm specifies the group the study of which leads to the description of the disease? As noted above, choice of this will depend upon the current state of knowledge. When this choice has been made, quantitative terms for practical application of the definition are required. The norm for the selected characteristic must be ascertained, and then the magnitude of the deviation from it that will be accepted as significant must be decided.

The difficulty of these steps varies with the nature of the defining characteristic. It is least for diseases $\vec{F}$ defined aetiologically, for which the defining $\stackrel{\text { ? }}{?}$ characteristic is categorical; though even here decision may be required about the magnitude of effects of the causal factor that will be regarded as indicative of disease. For diseases defined in terms of abnormal structure or function, dimensional factors are required is also in the specification of the defining characteristic. $\vec{A}$ In scientific work, specification of these factors may require appropriate statistical treatment of data $\vec{\omega}$ derived from study of relevant populations; in clinical practice, simple criteria, such as association with $\underset{\mathbb{\Phi}}{\overrightarrow{0}}$ relevant symptoms and signs, usually suffice.

\section{Factual and evaluative elements}

In this discussion, I shall use 'factual' to mean capable $\overrightarrow{\vec{\infty}}$ of being stated in objectively demonstrable terms, 음 preferring it to 'descriptive', because I have used the phrase 'clinical description' and wish to consider how $\mathcal{C}$ far clinical description can be objective. 'Evaluative' will be taken to mean involving value judgements; in these, things are assessed on a scale of goodness and badness, for which criteria are required.

I distinguish between two sorts of evaluative criterias $\bar{\phi}$ objective, which can be stated in terms of factua comparisons with specified norms available to general. inspection, and in principle quantifiable; ang subjective, which cannot be stated in such terms.

The general statement seeks to maximise the factual content in definitions of diseases for use in scientific contexts, eliminating elements that are not objectively demonstrable wherever possible. How far does it succeed? This question can be considered in relation to two elements in the statement: 1) defining characteristics and 2) biological disadvantage.

1) Defining characteristics can be stated factually for $\overparen{\overparen{D}}$ all diseases defined aetiologically or by disorders of $\dot{0}$ function or structure and for those defined syndromally in which the description of the syndrome includes objectively observable elements. For some of those defined syndromally, symptoms constitute the 옥 principal part of the defining characteristics; these $>$ include many in the field of psychiatry, especially the common affective disorders. With this exception, N which is discussed below, the demonstration of defining characteristics does not involve value judgements.

2) The proviso 'in such a way as to place them at a biological disadvantage', which is required to 0 distinguish the sort of deviation from the norm that $\bar{\Phi}$ will be accepted as indicative of disease from one that is $\stackrel{\odot}{+}$ regarded as harmless, is evidently evaluative, but can in principle be applied objectively in general biological contexts. Assessment of disadvantage requires $\stackrel{D}{\overparen{D}}$ comparison of those having the defining characteristic $\stackrel{\mathbb{Q}}{\propto}$ with members of the same species not showing it and $\bar{\sigma}$ living in a similar environment; if quantifiable criteria 
can be specified, this comparison will become factual. In human medicine, disadvantage in terms of inability to undertake normal activities or threat to life is obvious for most 'organic' diseases. In other instances, agreement about objectively demonstrable criteria by which disadvantage may be assessed should resolve controversies about whether a specified group should be said to be suffering from a disease.

The assessment of 'disadvantage' in relation to the whole group having the defining characteristic rather than to the individual resolves several problems:

(a) It avoids quibbles based on contingent circumstances of individuals, such as the soldier for whom a skin disease may diminish the risk of death in battle.

b) It makes it permissible to say that someone has a symptomless disease. Most people over middle-age have symptomless atherosclerosis. This is properly regarded as a disease, since as a group those with it have a shorter life expectancy than those who do not, although many individuals will suffer no evident illeffects from it and live to die of some other disease.

c) It clarifies discussion of some more general questions, such as whether heterozygosity for the sickle-cell gene should be considered a disease in areas where falciparum malaria is endemic. Homozygotes, carrying this gene on both chromosomes of pair 11, suffer severe symptoms from anaemia and a variety of changes in internal organs, leading to early death. Heterozygotes, carrying it on only one of this pair, do not suffer these ill-effects, but they differ from individuals not carrying it in two respects. They suffer from the disadvantage that if they mate with another heterozygote one in four of their offspring will suffer from sickle-cell anaemia; on the other hand, they have the advantage of being somewhat more resistant to the ill-effects of infection with $P$ falciparum. The balance between these can be discussed for the temporal and local circumstances.

I conclude that the general statement makes it possible to define most diseases in current nosology in factual terms, without subjective value judgements. This may not be possible for some psychiatric disorders currently definable only by symptoms; but in such instances, as discussed below, advances in knowledge may reduce or eliminate the subjective element.

\section{Some consequences of the general definition}

The general statement originated from an analysis of usages of the names of diseases whose purpose was to clarify medical discourse. It evidently has wider implications. Space permits only brief mention of these and some of them lead into fields in which I can claim no special expertise.

\section{SYNDROME, DISEASE, 'CLINICAL ENTITY'}

Questions such as 'Disease or syndrome?' and 'Is this a clinical entity?' are in effect requests for statement of the current basis of definition of a category. They probably arise from a feeling that a disease ought to be definable eventually, even if not now, in causal terms. But, as has been shown, the names of diseases defined in other ways are current in medical discourse, which without them would be intolerably prolix; and with due care they can be used clearly. One can speak of the syndrome of any disease with which a consistently recognisable pattern of symptoms and signs is associated. Whether a category definable only in clinical-descriptive terms is called a syndrome or a disease does not matter, provided that verbal usages are made explicit and applied consistently. 'Clinical entity' always needs ad hoc explanation; it often seems to be the refuge of one who has not succeeded in clarifying his or her thoughts, but is nevertheless determined to put them into words.

\section{PSYCHIATRIC NOSOLOGY}

Most psychiatric diagnostic categories, especially the affective disorders that are so prevalent, are definable only in syndromal terms. I suggest that there are two main causes for dissension about the validity of the concept of mental illness (14).

One is doubt about the possibility of an agreed objective basis for the elucidation of the symptoms which constitute the defining characteristics of such syndromes. The only evidence may be what the patient and those around him or her say about feelings and behaviour. Interpretation of some of this evidence requires value judgements for which it may be difficult to specify objectively demonstrable criteria. It is legitimate to hope that appropriate community studies may resolve this problem (15).

The other is failure to appreciate that in psychiatry, as in medicine generally, recognition of a syndromally defined category is to be regarded as the starting-point for advance towards its supersession by one or more categories defined in more precise terms, such as specific causal factors or disorders of function or of structure (6).

\section{'HOLISTIC MEDICINE'}

The general statement underlines the fact that diseases have no existence apart from that of the patients who are said to suffer from them. Acceptance of this makes medicine necessarily holistic. It is the patient whom we treat, not the disease. When we speak of the treatment of a disease, we must recognise that this phrase is an ellipsis for treatment of patients with that disease.

\section{DIAGNOSIS AND TREATMENT}

The general statement aims to maximise the factual content of medical diagnosis, minimising the need for value judgements, and providing a framework within which objective criteria for such judgements can be specified. Decisions about treatment, on the other hand, necessarily involve value judgements. The idea that medical practice proceeds from diagnosis to the treatment specified for the diagnosed disease by 
textbooks and other authorities is foolishly simplistic. 'Treatment' refers to a wide range of actions with differing objectives, and the possibilities vary according to the basis of definition of different diseases. For all patients, explanation, supportive advice, and measures for relief of symptoms are possible; for those with disorders of structure or of function, correction or amelioration may be possible; and for those with diseases of known cause, it may be possible to remove or neutralise the causal factor. The doctor needs factual knowledge of these possibilities, and of the risks that some of them carry. The application of this knowledge will be affected to varying degrees by the circumstances and feelings of the patient, and thus calls for value judgements of varying difficulty. The old aphorism 'Guérir quelquefois, soulager souvent, consoler toujours' recognises both the varying possibilities that may be available, and the duty to apply them to the greatest benefit of the patient.

Thus the realities of medical practice are far removed from the pattern underlying Hare's argument about mental illness (16). All arguments based upon a supposed 'medical model of disease' are suspect, since current nosology includes diseases defined by criteria of several different sorts, having different factual implications.

\section{THE DEFINITION OF HEALTH}

Professor Hare suggests that the concept of health is necessarily evaluative. He criticises Boorse's (17) attempt to define it in entirely descriptive terms.

As a physician, I am content to define health as the absence of disease. In this, I am in apparent agreement with both Boorse and Hare. I agree also with Boorse's trenchant criticism of concepts of 'positive health'. If someone asks 'Am I healthy?', all the doctor can do is to seek for evidences of known diseases and for significant deviations of structure and function from expected norms, and reassure the enquirer if he finds none. The evaluative element in 'health', so defined, is thus similar to that in definitions of diseases, from which, with the exception of some syndromally defined categories in psychiatry, the general statement permits evaluation not based on objectively demonstrable criteria to be eliminated.

Boorse seeks to exclude subjective value judgements from concepts of health and disease by defining diseases as internal states that depress functional activity below species-typical levels; health and disease are related to norms which can be established by appropriate statistical studies. While I am in sympathy with his aim to make the concepts of health and disease, certainly for use in scientific contexts, as free as possible from subjective value judgements, I am doubtful about several points in his analysis. As criteria distinguishing disease from health, depression of functional activity, which Boorse adopts, and biological disadvantage, used in the general statement, can both be applied objectively. But I think that
Boorse's definition gives undue prominence to functional deficit as a defining characteristic of diseases, and thus conflicts with current nosology, in which diseases are defined by several sorts of characteristics, among which aetiology takes precedence. For this reason, I prefer the wider criterion of biological disadvantage to distinguish deviations from the norm that should be accepted as defining characteristics of diseases. The identification of disease with an 'internal state' is confusing. This state is presumably regarded as the explanation of the phenomena constituting a disease; but at the same time, it is itself implied from these phenomena. In fact, the level to which we can take the explanatory process varies for different diseases; a state of affairs reflected in the general statement, which allows the names of diseases to have different sorts of causal implication.

The general statement about the proper use of the names of diseases is intended to make it possible to express the end-point of the diagnostic process without subjective value judgements; the judgement implicit in saying that observed phenomena constitute a disease is related to biological disadvantage, which can, in principle, be assessed objectively. I think that it can be applied successfully in most medical contexts, though difficulties persist for some syndromally defined psychiatric categories.

It is a legitimate aim of medical science to free medical concepts of disease, and consequently of health, from value judgements not based on objectively? demonstrable and quantifiable criteria. On the other hand, decisions about the management of individual patients, including not only therapeutic but also complex investigative procedures, involve value judgements of varying difficulty, into which subjective elements necessarily enter.

\section{Appendix 1}

TUBERCULOSIS AS A MODEL FOR CHANGING MEDICAL CONCEPTS

Until 1882, patients who would now be said to be suffering from tuberculosis were placed in a number of different diagnostic categories; and diverse views were held about the causation of these and about their relationship, if any, to each other. Initially, they were described and defined in clinical-descriptive terms. Pulmonary consumption or phthisis was known from ancient times and named after the characteristic wasting with symptoms, such as cough and bloodspitting, referable to the lungs. Scrofula was recognised by chronic swelling of lymph-nodes in the neck; it was at one time called the King's Evil, because it was thought to be curable by the royal touch, providing an example, perhaps unique, of a disease named after its supposed cure. Caries of the spine was recognised by the deformity which it caused; its association with palsy of the legs was noted by Hippocrates, and fully described by Pott in 1779. Tabes mesenterica was recognised by swelling of the abdomen by enlarged 
mesenteric lymph-nodes and wasting. There was uncertainty and controversy about the causation of these syndromally-defined diseases and about the relationship between them.

The first objective evidence of a relationship between them was morbid-anatomical. From the middle of the 17 th century onwards small pale nodules, which were named tubercles, were recognised in affected tissues, and later these were found to have a characteristic microscopic appearance. By the early 19th century, this led to the possibility of re-definition in morbid-anatomical terms, with the new name 'tuberculosis', first suggested by Schönlein in 1839 . But this unification was not generally accepted; the great Virchow at first denied that phthisis and scrofula should be regarded as tuberculous and opposed the idea that lupus vulgaris, later shown to be a form of tuberculosis of the skin, was related to scrofula.

Views about their causation varied from attribution to evil spirits through the humoral theories of Hippocrates and of ancient Hindu writers and the Galenical theory of ulceration of the lungs by 'pituita' dropping from the head, to constitutional predisposition. Communicability by contagion was denied by some and asserted by others. There was no objective way of resolving these controversies until Villemin in 1867 showed experimentally that tuberculosis was communicable, and Koch in 1882 demonstrated in histologically tuberculous tissues a bacillus which could cause similar changes in artificially infected animals. After this discovery, the disease 'tuberculosis' could be re-defined in aetiological terms. This led to the unification of a number of diseases previously defined syndromally or morbid-anatomically into a single aetiologically defined category. At the same time, some patients who would in the past have been said to be suffering from the syndromally-defined diseases phthisis and scrofula were excluded from this new category when no evidence of infection with Koch's bacilli could be found in them. Later, the complex natural history of this infection was elucidated; it was found that many people became infected but only some showed evidence of disease of varied severity, site and outcome.

The story is not ended, for there is still argument about the definition of tuberculosis. There are two views. One would limit 'tuberculosis' to disease caused by one species of mycobacterium, that which causes the form of morbid-anatomically defined tuberculosis most frequent in man; this organism was originally called the human tubercle bacillus but is now named Mycobacterium tuberculosis. This definition leaves in limbo cases of disease that pathologists recognise as tuberculosis but which are found to be caused by another species of mycobacterium. The other is that tuberculosis should be recognised as a compound diagnostic category, defined in both morbidanatomical and aetiological terms; full diagnostic statement would include specification of the causal mycobacterium, though it might be accepted as a convention that when this was not stated the organism had been shown or was assumed to be the human tubercle bacillus (18).

The history of tuberculosis thus illustrates how the definitions of diseases, as used in medical discourse, have changed and continue to change with advances from recognition by clinical description to knowledge of causation. It also emphasises the importance of recognising that a disease defined causally is the effect of a complex interaction between the causal agent and the affected individual.

f $G$ Scadding MD FRCP is Emeritus Professor of Medicine at the University of London and is Honorary Consultant Physician, Brompton Hospital, and Hammersmith Hospital, London.

\section{References}

(1) Hare $\mathrm{R}$ M. Health. Fournal of medical ethics 1986;12:174-181.

(2) Eden M. In: Jacquez J A, ed. The diagnostic process. Ann Arbor, Mich: University of Michigan, 1964: 64.

(3) Scadding J G. Diagnosis: the clinician and the computer. Lancet 1967;2:887-882.

(4) Kendall R E. The role of diagnosis in psychiatry. Oxford: Blackwell, 1975.

(5) Scadding J G. The concepts of disease: a reply. Psychological medicine 1980;10:425-427.

(6) Scadding J G. Review of (14) in Psychological medicine 1982;12:207-208.

(7) Scadding J G. Principles of definition in medicine. Lancet 1959;1:323-325.

(8) Scadding J G. Meaning of diagnostic terms in bronchopulmonary disease. British medical journal 1963;2:14251430.

(9) Scadding J G. The semantics of medical diagnosis. Biomedical computing 1972;3:83-90.

(10) Scadding J G. Talking clearly about broncho-pulmonary diseases. In: Scadding J G, Cumming G, eds. Scientific foundations of respiratory medicine. London: Heinemann, 1981: Ch 2.

(11) Popper K R. The open society and its enemies. London: Kegan Paul, 1945: vol 2, ch 11.

(12) de Dombal F T, Leaper D J, Staniland J R, McCann A P, Horrocks J C. Computer-aided diagnosis of acute abdominal pain. British medical journal 1972;2:9-13. de Dombal F T, Horrocks J C, Walmsley G, Wilson P D. Computer-aided diagnosis and decision-making in the acute abdomen. Fournal of the royal college of physicians 1975;9:211-218.

(13) Campbell E J M, Scadding J G, Roberts R S. The concept of disease. British medical journal 1979;2:757762.

(14) Szasz T. The myth of mental illness. New York: Harper and Row, 1961.

(15) Wing J K, Bebbington P, Robbins L N, eds. What is a case? The problem of definition in psychiatric community surveys. London: Grant McIntyre, 1981.

(16) See reference (1): 179.

(17) Boorse C. Health as a theoretical concept. Philosophy of science 1977 ; 44:552-573.

(18) Scadding J G. Nomenclature of mycobacterial disease. American review of respiratory disease 1987 ; 136:13081309. 